\title{
Rare Clinical and Radiologic Case of Cholangiocarcinoma Mimicking Pyogenic Abscess, Hepatic Echinococcal Cysts, and Metastases
}

\author{
Si Hyeong Lee', Soo Hyung Ryu', Dong Hoon Lee', Won Eui Yoon', Tae Young Park', Hye Kyung Lee², \\ Jeong Seop Moon ${ }^{1}$ \\ Departments of ${ }^{1}$ Internal Medicine, ${ }^{2}$ Pathology, Seoul Paik Hospital, Inje University College of Medicine, Seoul, Korea
}

Received Apr. 21, 2020

Revised Jun. 5, 2020

Accepted Jun. 7, 2020
Cholangiocarcinoma is a biliary carcinoma with a wide spectrum of imaging, histological, and clinical features. In immunocompromised patients, pyogenic abscesses are relatively common and an echinococcal hepatic cysts are very rare. The authors experienced a very rare case of cholangiocarcinoma showing multiple hypodense masses with wall enhancement mimicking pyogenic liver abscess, echinococcal hepatic cyst, and cystic metastases. An 83-year-old man, complaining of fatigue and poor oral intake, presented to our outpatient clinic. Abdominal computed tomography (CT) revealed multiple, variable-sized hypodense masses with peripheral rim enhancement throughout the liver. Dynamic liver magnetic resonance images also showed findings similar to those of a CT scan. We performed ultrasound-guided biopsy of the mass which revealed cholangiocarcinoma. (J Liver Cancer 2020;20:173-176)

Keywords: Cholangiocarcinoma; Liver abscess; Echinococcus; Metastasis

\section{INTRODUCTION}

Cholangiocarcinoma is a biliary carcinoma that arises from the intra- and extra-hepatic bile ducts, with a typical growth pattern that can be classified as exophytic (mass-forming), infiltrative (periductal), polypoid (intraductal), or a combination of these. ${ }^{1}$ Most cholangiocarcinomas are ductal (well, moderately, or poorly differentiated) adenocarcinomas. Other histologic types of cholangiocarcinoma can also be seen with microscopy: papillary, mucinous, signet-ring cell, mucoepidermoid, adenosquamous, squamous and cystadenocarcinoma. ${ }^{1,2}$ Cholangiocarcinomas of unusual histologic type show a growth pattern different from that of typical

\section{Corresponding author : Soo Hyung Ryu}

Department of Internal Medicine, Seoul Paik Hospital, Inje University College of Medicine, 9 Mareunnae-ro, Jung-gu, Seoul 04551, Korea

Tel. +82-2-2270-0001, Fax. +82-2-2270-3012

E-mail; rshdrryu@hanmail.net

https://orcid.org/0000-0002-1654-7057 ones. Accordingly, cholangiocarcinoma has a wide spectrum of radiologic, histological, and clinical appearances that may overlap with those of other hepatobiliary diseases, including benign varieties. ${ }^{1}$ In the present case, the authors experienced a rare case of cholangiocarcinoma mimicking pyogenic liver abscess, echinococcal hepatic cyst, and cystic metastases. This case study was reviewed and approved by Seoul Paik Hospital Institutional Review Board (IRB number: 2020-05-002).

\section{CASE REPORT}

An 83-year-old man complaining of fatigue and poor oral intake visited our hospital. He presented with weight loss and fever of up to $38.6^{\circ} \mathrm{C}$. He had a medical history of cardiovascular disease and diabetes and had undergone cholecystectomy 5 years before due to acute cholecystitis with gall bladder perforation. The previous abdominal ultrasonography showed no abnormality in the liver. Laboratory data revealed 
hemoglobin of $8.3 \mathrm{~g} / \mathrm{dL}$, WBC of normal range, C-reactive protein of $15.2(0-0.8) \mathrm{mg} / \mathrm{dL}$, procalcitonin (which can be elevated in serious bacterial infections) of 1.89 (under 0.05) $\mathrm{ng} / \mathrm{mL}$, and albumin of 2.6 (3.5-5.2) g/dL. The results of tumor markers were $\alpha$-fetoprotein (AFP) of 18.2 (0-10) ng/mL, carbohydrate antigen 19-9 (CA19-9) of 88.3 (0-37) U/mL, and protein induced by vitamin $\mathrm{K}$ absence or antagonist-II (PIVKA-II) of 64 (under 40) mAU/mL.

Both hepatitis B surface antigen and surface antibody were negative. Hepatitis $\mathrm{C}$ antibody was also negative. Parasite specific antibody tests for Clonorchis sinesis, Paragonimus westermani, Cysticercus and Sparganum were all negative. Gram stain and blood culture showed no growth or staining of pathogenic organisms. An abdominal computed tomogra- phy (CT) revealed multiple variable-sized, hypodense masses with peripheral rim enhancement throughout the liver (Fig. 1A). Dynamic liver magnetic resonance images also showed similar findings compared to a CT scan (Fig. 1B). After 10 days of a combination therapy of intravenous antibiotics consisting of imipenem/cilastatin and metronidazole, an abdominal CT showed no regression of masses throughout the liver. The ultrasonography-guided, percutaneous needle gun biopsy of the liver, at the "right lobe, target-like appearing mass", revealed mainly coagulative necrotic \& minimally viable, malignant large round to polygonal cell neoplasm, poorly differentiated, with massive hepatic parenchymal invasion (Fig. 2). The viable tumor cells exhibited typical histologic triad of adenocarcinomatous differentiation, with acinar, trabecular
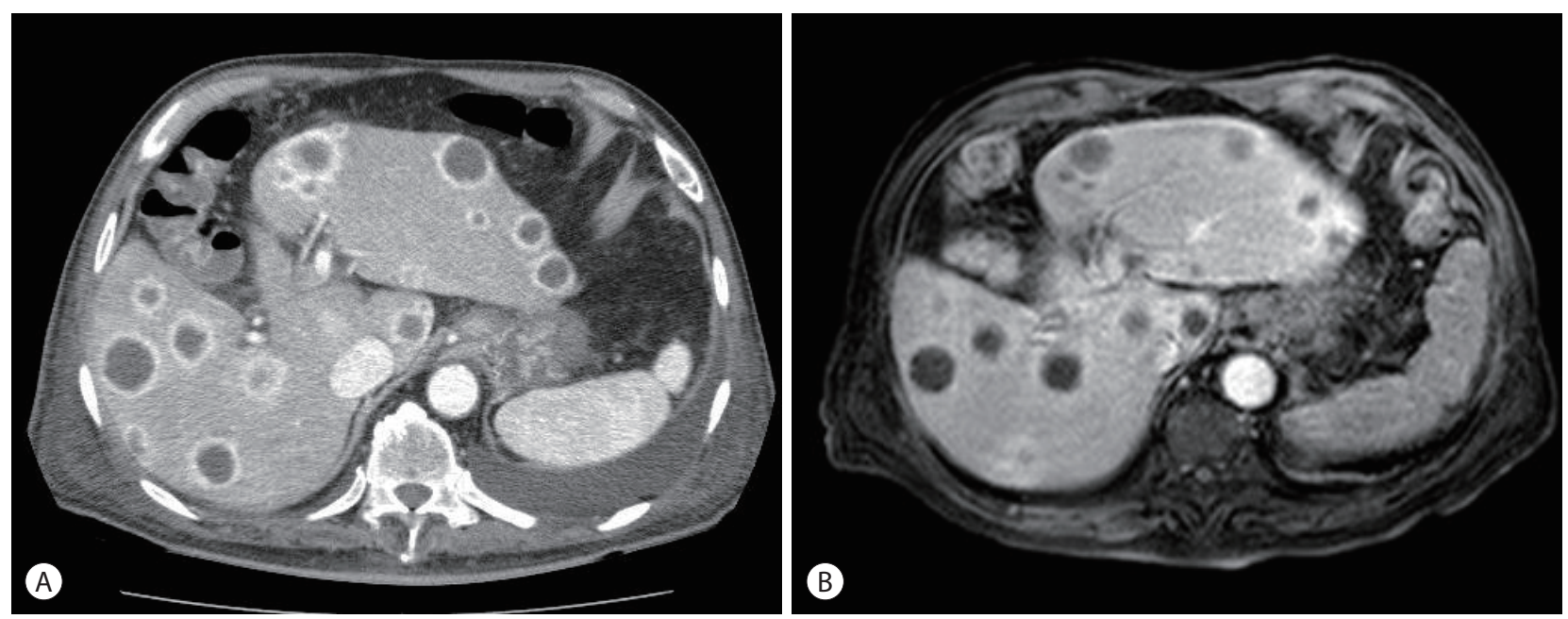

Figure 1. Imaging of the patient. (A) Enhanced computed tomography imaging of the arterial phase shows multiple hypodense masses with cystic components centrally and thin rim of peripheral enhancement. (B) Dynamic liver magnetic resonance images imaging reveals multiple round lesions measuring up to $3.5 \mathrm{~cm}$ with peripheral rim enhancement in the entire liver.
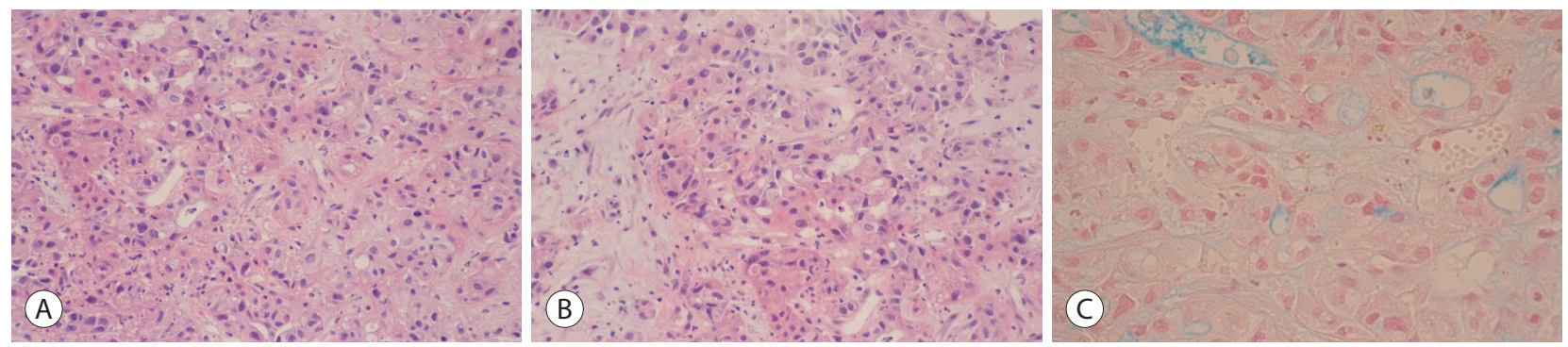

Figure 2. Histopathologic findings of fine needle aspiration biopsy specimen from liver mass. (A, B) The photomicrograph of the hepatic needle biopsy reveals malignant large round to polygonal cell neoplasm, poorly differentiated, with massive hepatic parenchymal invasion. (C) The viable tumor cells exhibit adenocarcinomatous differentiation, with acinar trabecular or solid sheet pattern of growth, moderately dense desmoplastic stroma and multifocal intracytoplasmic to luminal mucin production (hematoxylin and eosin, x200 [A, B]; Alcian blue staining, x400 [C]). 
or solid sheet pattern of growth (Fig. 2A), moderately dense desmoplastic stroma (Fig. 2B) and multifocal intracytoplasmic to luminal mucin production (Fig. 2C, Alcian blue stain). The tumor cells demonstrated immunohistochemical biliary differentiation, with immunoreactivities for CA19-9 (Fig. 3A) and cytokeratin 19 (Fig. 3B). The adenocarcinomatous differentiation was also showed by immunohistochemical reactivity for carcinoembryonic antigen (Fig. 3C). On the other hand, the tumor cells were not immunoreactive for $\mathrm{cy}$ tokeratin 20, thyroid transcription factor-1 and CD56. These morphological and immunohistochemical features strongly suggested the possibility of intrahepatic cholangiocarcinoma. As for the differential diagnosis, the possibility of hepatocellular carcinoma or metastatic adenocarcinoma from the lungs, the colorectum, the stomach or the non-pancreatobiliary system can be excluded. Despite combination antibiotic therapy and best supportive care, he died of heart failure 18 days after admission.

\section{DISCUSSION}

This case highlights the diagnostic difficulties of distinguishing intrahepatic cholangiocarcinoma from other liver diseases. Historically, pyogenic liver abscess caused by a bacterial infection in the liver parenchyma is usually caused by acute appendicitis, but with the development of surgical practice and microbiology, biliary tract disease is the most common identifiable cause. ${ }^{3}$ To our knowledge, few cases of intrahepatic cholangiocarcinoma mimicking liver abscess radiologically have been reported. ${ }^{4-6}$
Meanwhile, echinococcal hepatic cyst is a widely endemic helminthic disease, however, few cases have been reported in literature based on identification of the parasite in the Republic of Korea. Depending on the activity of the disease, serological tests may not be sensitive enough. ${ }^{7}$ Furthermore, images of echinococcal hepatic cysts resemble a variant type of hepatocellular carcinoma and intrahepatic cholangiocarcinoma, ${ }^{8}$ and up to $20-40 \%$ of patients have multiple cysts. ${ }^{9}$

The radiologic appearance of metastatic adenocarcinoma is one of the great mimics of exophytic peripheral cholangiocarcinoma, because metastatic adenocarcinoma in the liver is histologically the same as cholangiocarcinoma. ${ }^{1}$ Hepatic metastases are 18-40 times more common than primary liver tumors. ${ }^{10}$ Liver metastases are typically hypoattenuating on pre-enhanced CT, enhancing less than the surrounding liver following contrast.

Intrahepatic cholangiocarcinoma is a rare condition which typically occurs in males aged 50-70, and presents with symptoms related to biliary obstruction including jaundice, pruritus and dark urine. ${ }^{11}$ It usually appears as a solid mass showing low attenuation on pre-enhanced CT, ${ }^{12,13}$ however, it has a wide spectrum of radiologic, histological, and clinical appearances that may overlap with those of other hepatobiliary diseases. Essentially, diagnosis of intrahepatic cholangiocarcinoma still requires a combination of clinical, radiologic and nonspecific biochemical markers. ${ }^{14}$

In the present case, initial contrast enhanced CT showed multiple hypodense masses with central cystic components and thin rims of peripheral enhancement. This imaging feature could mimic various conditions in the liver. An abdomi-
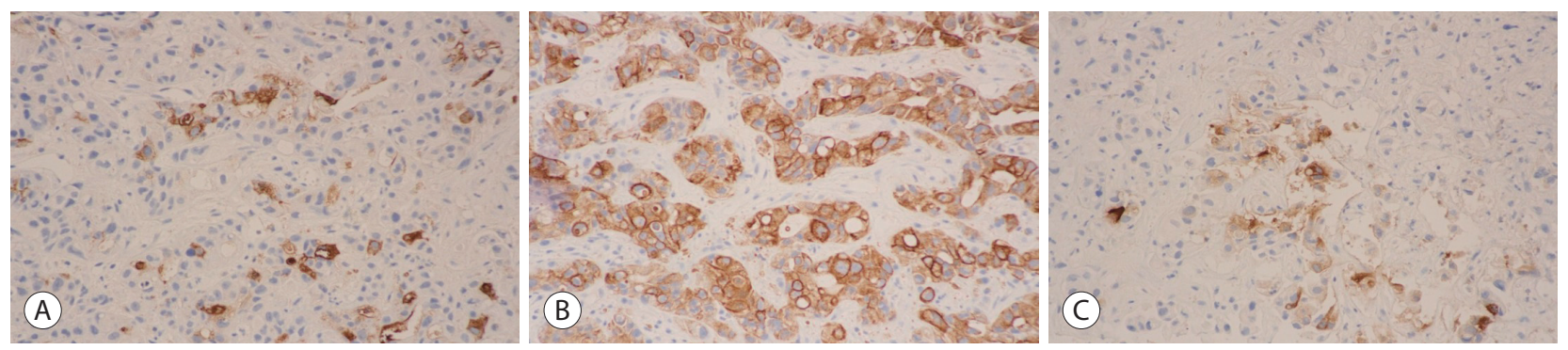

Figure 3. Immunohistochemical findings of fine needle aspiration biopsy specimen. The tumor cells demonstrate immunohistochemical biliary differentiation, with immunoreactivities for carbohydrate antigen 19-9 ( $A, x 200)$ and cytokeratin $19(B, x 200)$, together with adenocarcinomatous differentiation, with immunoreactivity for carcinoembryonic antigen $(C, \times 200)$. 
nal ultrasound scan performed 1 year ago showed no abnormality, which made it very difficult to assess a differential diagnosis between various lesions in the liver. Because the patient clinically progressed to sepsis, first, we considered the possibility of infectious disease, such as pyogenic liver abscess or echinococcal hepatic cysts, but blood cultures and parasite specific antibody tests were all negative. Despite broad spectrum antibiotics therapy with imipenem/cilastatin and metronidazole for 10 days, there was no improvement of the clinical course and radiological findings. There was no other primary malignancy indicated by the initial CT, and thus the second possibility is that primary liver cancer developed. Because of patient's poor general medical condition, upper and lower gastrointestinal endoscopy was not performed. The results of tumor markers were AFP of $18.2(0-10) \mathrm{ng} / \mathrm{mL}$, CA19-9 of 88.3 (0-37) U/mL, and PIVKA-II of 64 (under 40) $\mathrm{mAU} / \mathrm{mL}$. Because heterogeneous features of liver malignancy can sometimes mimic liver abscess, ultrasound-guided fine needle aspiration biopsy was performed to confirm the diagnosis. Eventually, the histological and immunohistochemical examinations concluded for features of cholangiocarcinoma, poorly differentiated. The authors experienced a rare case of intrahepatic cholangiocarcinoma mimicking pyogenic abscess, echinococcal hepatic cysts, and cystic metastases.

\section{Conflicts of Interest}

The authors have no conflicts of interest to disclose.

\section{REFERENCES}

1. Lee WJ, Lim HK, Jang KM, Kim SH, Lee SJ, Lim JH, et al. Radiologic spectrum of cholangiocarcinoma: emphasis on unusual manifestations and differential diagnoses. Radiographics 2001;21:S97-S116.
2. Nakajima T, Kondo Y, Mayazaki M, Okui K. A histopathologic study of 102 cases of intrahepatic cholangiocarcinoma: histologic classification and modes of spreading. Hum Pathol 1988;19:1228-1234.

3. Huang CJ, Pitt HA, Lipsett PA, Osterman FA Jr, Lillemoe KD, Cameron JL, et al. Pyogenic hepatic abscess. Changing trends over 42 years. Ann Surg 1996;223:600-607; discussion 607-609.

4. Rahimian J, Wilson T, Oram V, Holzman RS. Pyogenic liver abscess: recent trends in etiology and mortality. Clin Infect Dis 2004;39:16541659.

5. Kokubo T, Itai Y, Nagao T. Intrahepatic cholangiocarcinoma with cystic formation. Radiat Med 1990;8:219-221.

6. Kim KH, Cho OK, Kim YS, Rhim HC, Koh BH. Intrahepatic cholangiocarcinoma presenting as liver abscess: report of two cases. J Korean Radiol Soc 1998:39:773-777.

7. Pakala T, Molina M, Wu GY. Hepatic echinococcal cysts: a review. J Clin Transl Hepatol 2016;4:39-46.

8. Sade R, Kantarci M, Ogul H, Gundogdu B, Aydinli B. Differentiation between hepatic alveolar echinococcosis and primary hepatic malignancy with diffusion-weighted magnetic resonance imaging. Diagn Interv Imaging 2018;99:169-177.

9. Nunnari G, Pinzone MR, Gruttadauria S, Celesia BM, Madeddu G, Malaguarnera $G$, et al. Hepatic echinococcosis: clinical and therapeutic aspects. World J Gastroenterol 2012;18:1448-1458.

10. Namasivayam S, Martin DR, Saini S. Imaging of liver metastases: MRI. Cancer Imaging 2007;7:2-9.

11. Tasch JJ, Dube N. An unusual presentation of advanced intrahepatic cholangiocarcinoma: when biopsy results fail. Am J Case Rep 2018;19:35-40.

12. Lacomis JM, Baron RL, Oliver JH 3rd, Nalesnik MA, Federle MP. Cholangiocarcinoma: delayed CT contrast enhancement patterns. Radiology 1997;203:98-104.

13. Wibulpolprasert B, Dhiensiri T. Peripheral cholangiocarcinoma: sonographic evaluation. J Clin Ultrasound 1992;20:303-314.

14. Banales JM, Cardinale V, Carpino G, Marzioni M, Andersen JB, Invernizzi $P$, et al. Expert consensus document: cholangiocarcinoma: current knowledge and future perspectives consensus statement from the European Network for the Study of Cholangiocarcinoma (ENS-CCA). Nat Rev Gastroenterol Hepatol 2016;13:261-280. 